\title{
Choanal stenosis and atresia
}

S Moosa
MB ChB, BSc Hons (Pharm), MPhil,
FCRad (Diag)
Department of Radiology
Groote Schuur Hospital and University of Cape Town.
Y VadaChia
MB ChB
S AndronikOu
MB BCh, FCRad (D) (SA), FRCR (Lond)
Department of Radiology
Tygerberg Hospital and University of Stellenbosch

Stenosis or atresia of the posterior nasal cavity, i.e. the choanae, is the most common congenital abnormality of the nasal cavity. Stenosis is considered to be more common than atresia. Bilateral atresia is life-threatening in neonates, who are obligate nasal breathers. These patients present early with severe respiratory distress, aggravated by feeding. Other pointers to the diagnosis include the inability to pass a nasogastric tube and relief of respiratory compromise on crying. The patients with unilateral disease typically present later in life with unilateral, chronic suppurative rhinorrhoea. This form of the condition is more common but due to its chronic, relapsing symptomatology the diagnosis is often delayed. Definitive diagnosis can be made by computed tomography (CT) scanning. Using images from our practice, we would like to propose an approach to scanning and interpretation of this entity.

CT is the imaging modality of choice. Patients are examined supine, with nasal passages well suctioned before imaging commences. Topical nasal vasoconstrictors may be helpful to reduce mucosal swelling. The gantry is angled $5^{\circ}$ cephalad to a plane parallel to the hard palate. One to $1.5 \mathrm{~mm}$ thick uncontrasted scans are obtained contiguously. The skull base is only partially ossified at birth and therefore conventional bone-window settings may not show the bony margins. High-resolution bone filters help overcome this problem.

Interpretation of the images rests on measurements of the posterior vomer, the choanal openings and the shape of the posteromedial maxilla. The anatomical landmark at which to evaluate the scan is at the level of the pterygoid plates (Fig. 1). Below this level the hard palate is visualised and above this level structures within the nasal cavity make measurement difficult. At this level the width of both posterior choanae at maximum stenosis and the maximum width of the inferoposterior vomer are measured. The vomer is widened in most cases. In patients less than 8 years of age the mean vomer width is $2.8 \mathrm{~mm}$ and should not exceed $5.5 \mathrm{~mm}$. The mean width of the posterior choanal airspace in the neonate is $6.7 \mathrm{~mm}$, increasing to $8.6 \mathrm{~mm}$ at 6 years and $11.3 \mathrm{~mm}$ by age $16 .^{1,2}$
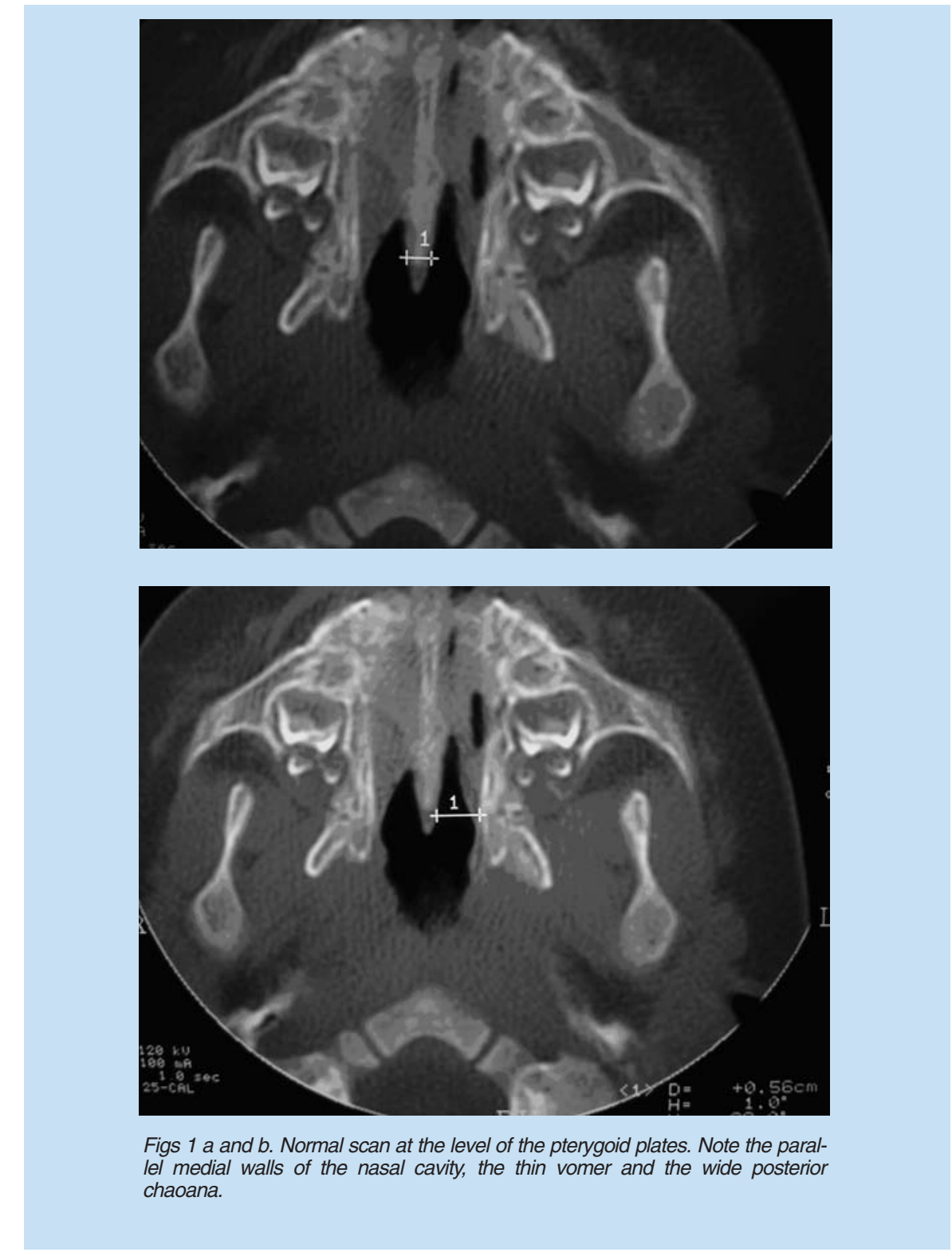

Figs $1 \mathrm{a}$ and $b$. Normal scan at the level of the pterygoid plates. Note the parallel medial walls of the nasal cavity, the thin vomer and the wide posterior chaoana. 


\section{PICTORIAL INTERLUDE}

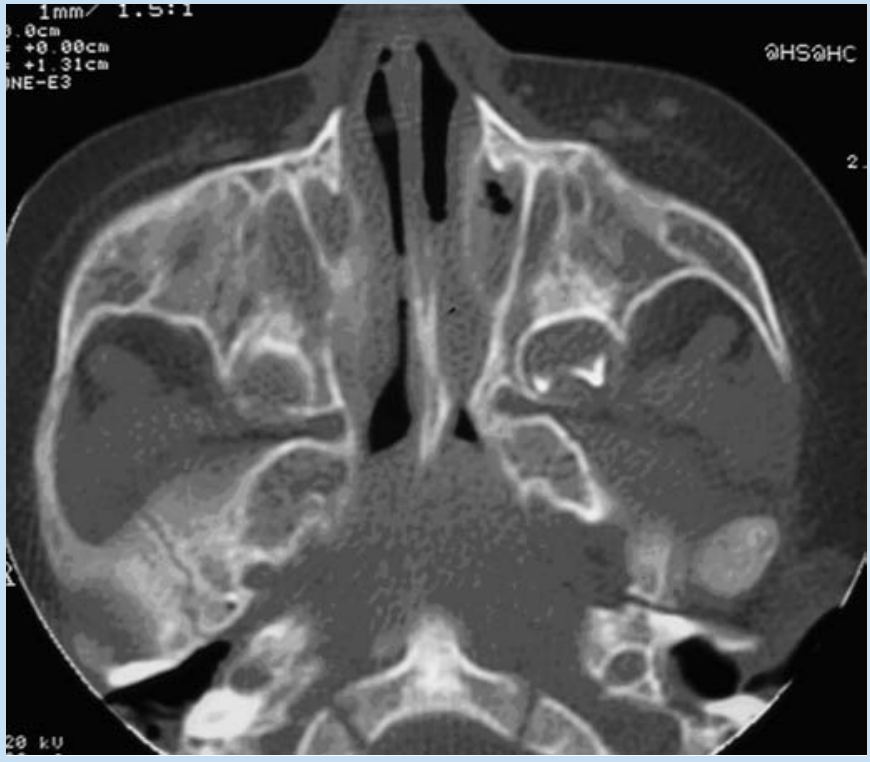

Fig. 2. CT scan showing unilateral left-sided stenosis with unilateral thickening of the vomer and medially bowed left medial maxillary wall.
The normal posteromedial maxilla is straight and parallel. In choanal stenosis/atresia, it is typically medially bowed and may touch the lateral extent of the vomer (Fig. 2). Membranous atresias will show soft-tissue densities in the posterior choanae and narrowing of the posterior choanae just anterior to the pterygoid plates. In the case of bony atresia, the thickness of the atretic plate should be reported for surgical planning.

Patients require surgical treatment, which is urgent in the case of bilateral atresia. CT evaluation of suspected choanal atresia/stenosis confirms the diagnosis and helps plan surgery. Measurements of the structures outlined allows for an objective and reproducible set of criteria for the diagnosis of choanal atresia and stenosis.

\section{References}

1. Slovis TLR. Choanal atresia: precise CT evaluation. Radiology 1985; 155: 345-348.

2. Hudgins PA, Jacobs IN, Castillo M. Pediatric airway disease. In: Som PM, Curtin HD, ed. Head and Neck Imaging. Philadelphia: Mosby, 1996. 